\title{
Nutritional and immune status following spinal cord injury: a case controlled study
}

\author{
AC Lynch ${ }^{1}$, C Palmer ${ }^{1}$, AC Lynch ${ }^{2}$, A Anthony ${ }^{2}$, JA Roake ${ }^{1}$, J Frye ${ }^{1}$ and FA Frizelle*, \\ ${ }^{1}$ Colorectal Unit, Department of Surgery, Christchurch Hospital; Christchurch, New Zealand; ${ }^{2}$ Spinal Injuries Unit, \\ Burwood Hospital, Christchurch, New Zealand
}

Study design: Case controlled study.

Objective: To compare nutritional status and immune response in a group of spinal cord injured (SCI) patients with age and gender matched non SCI control subjects.

Method: Thirty past patients of the Burwood Hospital Spinal Injuries Unit living locally were enrolled in the study. Age and gender matched non SCI control subjects were selected volunteers from hospital staff. Nutritional status was assessed by generating a Nutritional Risk Score (NRS, Appendix 1) and drawing blood for full blood count, iron studies, red blood cell folate, vitamin B12, ferritin, magnesium, and zinc. Immune status was assessed by vaccination response index (VRI) to Pneumovax 23 vaccine.

Results: Full blood count, iron studies, and testing for red blood cell folate, albumin, prealbumin, vitamin B12, ferritin, magnesium and zinc were normal range for both groups. The SCI group had significantly different median values than controls $(P<0.01)$ for haemoglobin concentration, white blood cell count, albumin, prealbumin, serum iron and $\%$ saturation. Body Mass Index (weight $\mathrm{kg} /\left(\right.$ height $\mathrm{cm}^{2}$ ) was 22.2 (range 15-30) for the SCI group, significantly less than the paired control group index of 26 (range $20-32, P=0.0004$ ). Median NRS for SCI patients was 2 (range $0-6$ ), compared to 0 (range 2-4) for paired controls $(P<0.0001)$. Scores ranged from 0 to 2 for each of the five NRS components for the SCI patients and 0 to 3 for the control group. There was no significant difference in the preand post-vaccination ratio for $\operatorname{IgG}, \operatorname{IgA}$, and $\operatorname{IgM}$ response to Pneumovax 23 vaccine.

Conclusion: We have not identified any nutritional or immune status abnormality in SCI patients, however the SCI patients have a lower value for certain nutritional parameters and BMI. SCI patients however are at only slight risk of nutritional problems given their NRS and their lower normal values for certain nutritional factors.

Spinal Cord (2002) 40, 627-630. doi:10.1038/sj.sc.3101382

Keywords: spinal cord injury; immune status; nutrition

\section{Introduction}

Wound healing can be a problem in SCI patients following major surgery. While this may be due to many reasons such as the lack of pressure sensation allowing undue pressure on wounds, we were concerned about the possibility of nutritional and or immune problems following SCI that may have an effect on wound healing. Our aim was to attempt to identify and quantitate differences in nutritional risk, nutritional proteins, and immunological response to vaccination between SCI

*Correspondence: FA Frizelle, Colorectal Unit, Department of Surgery, Christchurch Hospital, Riccarton Avenue, Christchurch, New Zealand patients and age and gender matched control subjects.

\section{Method}

Thirty past patients of the Spinal Injuries Unit, Burwood Hospital, Christchurch, New Zealand were identified and enrolled in the study. The patients had no intercurrent illnesses other than the known stable SCI. Age and gender matched controls were selected from non SCI hospital staff. Permission was obtained from the SCI participants to retrieve data relating to their injury from hospital case notes. All SCI patients were otherwise well, all were injured more than one year previously and had no current problems such as chronic pressure ulcers. 
Nutritional status was assessed by generating a Nutritional Risk Score (NRS, Appendix $1^{1,2}$ and drawing blood for full blood count, iron studies, red blood cell folate, vitamin B12, ferritin, magnesium, and zinc. Immune status was assessed by vaccination response index (VRI) to Pneumovax 23 vaccine. The vaccine was administered intramuscularly. The post vaccination specimen was taken 14 days post operatively in all cases.

Informed consent was obtained from all participants prior to enrolment, drawing of blood and administration of Pneumovax. The study had ethical approval from the Canterbury Ethics Committee, Christchurch. Non parametric statistical analysis was undertaken to be Wilcoxon test (paired). Significance was assumed at $(P<0.05)$.

\section{Results}

The mean age of the SCI participants was 44 years (range 22-67 years), four female and 26 male, and were matched with age, gender matched controls). Twenty SCI patients had cervical injuries (C3-C7, 10 SCI patients had thoracic injuries (T11-T12).

Full blood count, iron studies, and testing for red blood cell folate, total protein, albumin, prealbumin, vitamin B12, ferritin, magnesium and zinc was performed. Median results for each group and normal ranges are presented in Table 1. Results were not outside the normal laboratory range for either group, however the SCI group had significantly different median values than controls $(P<0.01)$ for haemoglobin concentration, white blood cell count, albumin, prealbumin, serum iron and \% saturation.

\section{Body mass index}

Median weight for SCI participants was $65.5 \mathrm{~kg}$ (range $50-93 \mathrm{~kg}$ ) compared to $80 \mathrm{~kg}$ (range $60-108 \mathrm{~kg}$ ) for the control group. Mean body mass index (weight $\mathrm{kg}$ / (height $\mathrm{cm}^{2}$ ) 22.2 (range 15-30) for the SCI group, significantly less than the paired control group BMI of 26 (range $20-32, P=0.0004$ ).

\section{Nutritional risk score}

Median NRS for SCI patients was 2 (range 0-6), compared to 0 (range $2-4$ ) for paired controls. There is a significant difference in NRS between groups: $P<0.0001$. Scores ranged from 0 to 2 for each of the five NRS components for the SCI patients and 0 to 3 for the control group.

Table 1 Median values and normal range for blood parameters measured in SCI patients $(n=30)$ and age and gender matched controls $(n=30)$

\begin{tabular}{|c|c|c|c|c|}
\hline Test & Normal range & $S C I$ & Controls & $\mathrm{P}$ value \\
\hline Haemoglobin & $135-175 \mathrm{~g} / 1$ & $\begin{array}{c}136 \\
(103-174)\end{array}$ & $\begin{array}{c}145 \\
(124-159)\end{array}$ & $0.004^{\mathrm{a}}$ \\
\hline White blood cell count & $4.0-11.0 \times 10^{9} / 1$ & $\begin{array}{c}6.8 \\
(4.3-12.6)\end{array}$ & $\begin{array}{c}5.9 \\
(2.1-7.7)\end{array}$ & $0.007^{\mathrm{a}}$ \\
\hline Red blood cell folate & $380-1200 \mathrm{nmol} / \mathrm{l}$ & $\begin{array}{c}644 \\
(366-982)\end{array}$ & $\begin{array}{c}589.5 \\
(361-1076)\end{array}$ & 0.33 \\
\hline Total protein & $60-80 \mu \mathrm{mol} / 1$ & $\begin{array}{c}72 \\
(65-89)\end{array}$ & $\begin{array}{c}76 \\
(71-84)\end{array}$ & 0.03 \\
\hline Albumin & $35-53 \mathrm{~g} / 1$ & $\begin{array}{c}38 \\
(32-44)\end{array}$ & $\begin{array}{c}42 \\
(36-46)\end{array}$ & $0.0001^{\mathrm{a}}$ \\
\hline Prealbumin & $0.2-0.3 \mathrm{~g} / 1$ & $\begin{array}{c}0.26 \\
(0.18-0.35)\end{array}$ & $\begin{array}{c}0.3 \\
(0.2-0.4)\end{array}$ & $0.003^{\mathrm{a}}$ \\
\hline Vitamin B12 & $120-450 \mathrm{pmol} / 1$ & $\begin{array}{c}240 \\
(144-417)\end{array}$ & $\begin{array}{c}293 \\
(157-936)\end{array}$ & 0.12 \\
\hline Ferritin & $15-120 \mu \mathrm{g} / 1$ & $\begin{array}{c}84 \\
(14-500)\end{array}$ & $\begin{array}{c}112 \\
(8-267)\end{array}$ & 0.94 \\
\hline Magnesium & $0.6-1.2 \mathrm{mmol} / 1$ & $\begin{array}{c}0.8 \\
(0.6-0.9)\end{array}$ & $\begin{array}{c}0.8 \\
(0.7-1)\end{array}$ & 0.04 \\
\hline Zinc & $10.0-17.0 \mu \mathrm{mol} / \mathrm{l}$ & $\begin{array}{c}11.6 \\
(8.7-34.2)\end{array}$ & $\begin{array}{c}13.1 \\
(9.6-16.2)\end{array}$ & 0.14 \\
\hline Serum iron & $10-34 \mu \mathrm{mol} / 1$ & $\begin{array}{c}13 \\
(5-23)\end{array}$ & $\begin{array}{c}19 \\
(11-31)\end{array}$ & $0.0006^{\mathrm{a}}$ \\
\hline Transferrin & $1.8-3.4 \mathrm{~g} / 1$ & $\begin{array}{c}2.3 \\
(1.7-2.9)\end{array}$ & $\begin{array}{c}2.3 \\
(1.9-2.9)\end{array}$ & 0.56 \\
\hline$\%$ Saturation & $16-45$ & $\begin{array}{c}20.5 \\
(10-40)\end{array}$ & $\begin{array}{c}29 \\
(17-63)\end{array}$ & $0.003^{\mathrm{a}}$ \\
\hline
\end{tabular}

Those values with a significant difference between groups are marked ${ }^{\mathrm{a}}$ 
Table 2 Immunoglobulin response to Pneumovax 23 vaccine for SCI patients $(n=30)$ and age and gender matched controls $(n=30)$

\begin{tabular}{lccc}
\hline & Pre-vaccination (range) & Post-vaccination (range) & Ratio $($ Post/Pre) \\
\hline IgG normal range & $11-45$ & $73-874$ & $2.8-37$ \\
Controls & $69(23-359)$ & $366(50-2433)$ & $4.2(1.3-11.8)$ \\
SCI & $70(17-196)$ & $340(26-4297)$ & $4(1.3-165.3)$ \\
IgA normal range & $<8-76$ & $103-1378$ & $3-50$ \\
Controls & $26(8-237)$ & $694(103-2941)$ & $19(7.9-95.9)$ \\
SCI & $39(8-168)$ & $617(120-3555)$ & $15(3.3-299)$ \\
IgM normal range & $8-186$ & $42-1431$ & $1.8-22$ \\
Controls & $48(8-246)$ & $254(26-2213)$ & $4.2(1.4-31.6)$ \\
SCI & $68(23-211)$ & $254(56-1277)$ & $4(1.1-23.2)$ \\
\hline
\end{tabular}

No significant difference between the groups was seen

\section{Immunoglobulin response}

Response to Pneumovax 23 vaccine was assessed by calculating the pre/post vaccination ratio for $\operatorname{IgG}, \operatorname{IgA}$, and IgM (Table 2). There was no significant difference between SCI patients and the control group for any value, with all ratios lying within the normal laboratory ranges. There was no significant difference between SCI patients and controls, nor for cervical injury SCI patients compared with those with thoracic injuries.

\section{Discussion}

Our results show a significant difference in certain nutritional parameters between healthy SCI patients and age and sex matched controls. SCI patients are lighter and have lower nutritional index scores. There was a significant difference between the two groups, with SCI patients more likely to fall in the 'needs monitoring' (scores 4-5) group. Previous studies have suggested that indicators of nutritional function such as zinc and prealbumin have been shown to be low in SCI patients with pressure ulcers. ${ }^{3,4}$ None of the SCI patients in our study had pressure ulcers and median blood levels of zinc and prealbumin were inside the normal range.

There is some evidence for changes in immune function following $\mathrm{SCI}^{5}$ including an impaired lymphocyte proliferation response in patients with complete SCI when compared with neurologically intact controls. ${ }^{6}$ These changes may be mediated by the autonomic nervous system and be dependent upon the neurological level. ${ }^{6}$ We found no significant difference in the ability of the SCI patients to mount an antibody response to the Pneumovax vaccine when compared to normal controls and did not demonstrate any association of strength of response with neurological level. This is consistent with responses to the trivalent 1994-1995 influenza vaccine reported previously. ${ }^{7}$

Pneumococcal vaccine responses have been shown to be impaired in immunocompromised patients in a variety of clinical settings. ${ }^{8-10}$ The implication of the Pneumovax response is that in healthy SCI patients both the afferent (antigen processing and presentation) and efferent (effector) limbs of the immune system remain globally intact. This appears to include the ability to mount both $\mathrm{T}$-independent responses (i.e. the IgM response to pneumococcal polysaccharides) and T-dependent (IgG and IgA) responses. However, subtle changes in immune cell function and $\mathrm{IgG}$ isotype secretion have not been excluded by this simple study.

Screening SCI patients for nutritional risk factors is unlikely to be useful to identify those with nutritional problems unless there is a clinical suggestion of a problem. In this situation addressing nutritional problems early may be useful in reducing the incidence of complications such as pressure ulcers. Further work could be done to investigate the relationship of impaired nutrition to immune function in SCI patients.

\section{Acknowledgements}

Christchurch School of Medicine Summer Studentship provided funding for salary for $\mathrm{C}$ Palmer. Funding for salary for AC Lynch was provided by the Canterbury Colorectal Research Fund. Expenses were paid by the Canterbury Colorectal Research Fund.

\section{References}

1 Wolinsky FD et al. Further assessment of the reliability and validity of a Nutritional Risk Index: analysis of a three-wave panel study of elderly adults. Health Serv Res 1986; 20: $977-990$.

2 Schneider SM, Hebuterne X. Use of nutritional scores to predict clinical outcomes in chronic diseases. Nutr Rev 2000; 58: $31-38$.

3 Cruse JM et al. Facilitation of immune function, healing of pressure ulcers, and nutritional status in spinal cord injury patients. Exp Mol Pathol 2000; 68: 38 - 54. 
4 Cruse JM et al. Review of immune function, healing of pressure ulcers, and nutritional status in patients with spinal cord injury. J Spinal Cord Med 2000; 23: 129-135.

5 Campagnolo DI, Bartlett JA, Keller SE. Influence of neurological level on immune function following spinal cord injury: a review. J Spinal Cord Med 2000; 23: $121-$ 128.

6 Campagnolo DI et al. Alteration of immune system function in tetraplegics. A pilot study. Am J Phys Med Rehabil 1994; 73: $387-393$.

7 Darouiche RO, Atmar R, Markowski J. Antibody response to influenza vaccination in spinal cord injury patients. Arch Phys Med Rehabil 1995; 76: 1032 - 1033.

\section{Appendix 1: Nutritional Risk Score}

\section{Unintentional weight loss in last 3 months}

No weight loss

$$
\begin{aligned}
& 0-3 \mathrm{~kg} \\
& >3-6 \mathrm{~kg} \\
& >6 \mathrm{~kg}
\end{aligned}
$$

Body Mass Index

$>20$

$18-19$

$15-17$

$<15$

\section{Appetite}

Good, most of 3 meals/day

Poor, leaving $>$ half of provided meals

Nil, unable to eat

\section{Ability to eat and retain food}

No difficulties, independent eating, no diarrhoea, no vomiting

Problems handling food, vomiting, mild diarrhoea

Difficulty swallowing, problems with chewing, slow to eat, needs help with eating, moderate vomiting/diarrhoea

1

Unable to take food orally, unable to swallow, malabsorption, severe vomiting/diarrhoea
8 French $\mathrm{N}$ et al. 23-valent pneumococcal polysaccharide vaccine in HIV-1 infected Ugandan adults: double blind, randomised and placebo controlled trial. Lancet 2000; 355: $2106-2111$.

9 Hartkamp A et al. Antibody response to pneumococcal and haemphilius vaccinations in patients with B-cell chronic lymphatic leukaemia. Vaccine 2001; 19: $1671-$ 1677.

10 McCashland TM, Preheim LC, Gebntery MJ. Pneumococal vaccine response in cirrhosis and liver transplantation. $J$ Infect Dis 2000; 181: 757-760.

\section{Stress factors}

Nil

Mild, minor surgery, minor infection

Moderate, chronic disease, major surgery, fracture, pressure area, inflammatory bowel disease, small bowel resection

Severe, multiple injuries, fractures or burns, malignancy, severe sepsis

Score

0-3 Low risk

4-5 Needs monitoring

$6-15$ High risk 